\title{
Pregnancy suppresses neuropathic pain induced by chronic constriction injury in rats through the inhibition of TNF- $\alpha$
}

This article was published in the following Dove Press journal:

Journal of Pain Research

8 March 2017

Number of times this article has been viewed

\section{Yoshiko Onodera \\ Megumi Kanao-Kanda \\ Hirotsugu Kanda \\ Tomoki Sasakawa \\ Hiroshi Iwasaki \\ Takayuki Kunisawa}

Department of Anesthesiology and Critical Care Medicine, Asahikawa Medical University, Hokkaido, Japan
Correspondence: Hirotsugu Kanda Department of Anesthesiology and Critical Care Medicine, Asahikawa Medical University, Midorigaokahigashi 2-I-I-I, Asahikawa, Hokkaido, 078-85I0, Japan

$\mathrm{Tel}+8 \mathrm{I} 166682583$

Fax +8I 166682589

Email h.kanda0629@nifty.com
Purpose : Pregnancy-induced analgesia develops during late pregnancy, but it is unclear whether this analgesia is effective against neuropathic pain. The detailed molecular mechanisms underlying pregnancy-induced analgesia have not been investigated. We examined the antinociceptive effect of pregnancy-induced analgesia in a neuropathic pain model and the expression of tumor necrosis factor (TNF)- $\alpha$, glial fibrillary acidic protein (GFAP), Iba-1, and c-Fos in the spinal dorsal horn just before parturition.

Materials and methods: Female Sprague Dawley rats (200-250 g) were randomly assigned to one of four groups (pregnant + chronic constriction injury [CCI]; pregnant + sham injury; not pregnant $+\mathrm{CCI}$; and not pregnant + sham injury). Separate groups were used for the behavioral and tissue analyses. CCI of the left sciatic nerve was surgically induced 3 days after confirming pregnancy in the pregnancy group or on day 3 in the not pregnant group. The spinal cord was extracted 18 days after CCI. TNF- $\alpha$, GFAP, Iba-1, and c-Fos expression levels in the spinal dorsal horn were measured by Western blot analysis. Mechanical threshold was tested using von Frey filaments.

Results: The lowered mechanical threshold induced by CCI was significantly attenuated within 1 day before parturition and decreased after delivery. TNF- $\alpha$ expression in CCI rats was decreased within 1 day before parturition. Further, GFAP, Iba-1, and c-Fos expression in the spinal dorsal horn was reduced in the pregnant rats. Serum TNF- $\alpha$ in all groups was below measurable limits. Conclusion: Our findings indicate that pregnancy-induced analgesia suppresses neuropathic pain through reducing spinal levels of TNF- $\alpha$, GFAP, Iba-1, and c-Fos in a rat model of CCI.

Keywords: pregnancy-induced analgesia, $\mathrm{TNF}-\alpha$, neuropathic pain, glial cell

\section{Introduction}

Neuropathic pain is caused by dysfunction of the peripheral nerves or, less frequently, the central nervous system. ${ }^{1}$ Neuropathic pain affects $7 \%-8 \%$ of the population, ${ }^{2}$ and is one of the most difficult-to-treat chronic pain states because it often does not respond to conventional analgesic therapies. ${ }^{3,4}$ Non-steroidal anti-inflammatory drugs are generally not effective for neuropathic pain. ${ }^{5}$ While opioids have demonstrated efficacy in clinical trials, they are not usually considered first-line therapy due to concerns regarding addiction and relatively poor adverse-effect profiles. ${ }^{6}$ While antidepressants and anticonvulsants are mainstay pharmacologic treatments, even these drugs have limited efficacy. ${ }^{7}$ Unfortunately, this maladaptive and unrelenting pain remains difficult to alleviate.

A previous report revealed significant changes in the response of rats to several forms of noxious stimulation, such as colorectal distention, on day 21 of gestation. ${ }^{8}$ 
In addition, many animal studies suggest that pregnancy modulates the endogenous pain control system and decreases pain thresholds. ${ }^{9-11}$ Some studies report that women experience a progressive increasing heat pain threshold during pregnancy, ${ }^{12}$ whereas others have not observed this phenomenon. ${ }^{13,14}$ Pregnancy-induced protection mechanisms against neuropathic pain have not been explored.

The proinflammatory cytokine tumor necrosis factor (TNF)- $\alpha$ is implicated in the pathogenesis of peripheral neuropathy. Epineural application of TNF- $\alpha$ elicits acute mechanical hyperalgesia beginning 1-3 days post-injection. ${ }^{15}$ TNF- $\alpha$ in the spinal dorsal horn regulates neuropathic pain induced by HIV-1 gp120 application in rats. ${ }^{16}$ Expression of TNF- $\alpha$ increases in the dorsal horn after spinal cord injury. ${ }^{17}$ Following chronic constriction injury (CCI), TNF- $\alpha$ increases in the dorsal horn. ${ }^{18}$ It is unclear, however, whether the CCIinduced TNF- $\alpha$ secretion is altered during pregnancy.

Surprisingly, to our best knowledge, few published studies have addressed possible mechanisms of pregnancy-induced analgesia using molecular biology techniques. We hypothesized that pregnancy-induced analgesia reduces neuropathic pain by inhibiting TNF- $\alpha$, which is produced from glial cells in the spinal dorsal horn. In this study, we examined the antinociceptive effect of pregnancy-induced analgesia in a rat model of CCI. We also analyzed the expression of some proteins associated with spinal glial activation in neuropathic animals by Western blot to assess their potential association with neuropathic pain. The aims of our study were to investigate the antinociceptive effect of pregnancy-induced analgesia in a neuropathic pain model and to measure the expression of TNF- $\alpha$ in the spinal dorsal horn just before delivery.

\section{Materials and methods}

\section{Animals}

All experimental procedures and housing conditions were approved by the Animal Research Committee (approval number: 16142, March 24, 2016) institutional animal care guideline of Asahikawa Medical University were followed. The study was conducted in accordance with the ethical guidelines of the International Association for the Study of Pain. ${ }^{19}$ Female Sprague Dawley rats ( 11 weeks old, 200-250 g) were housed 1-3 per cage for approximately 10 days before beginning the study. After mating or surgery, the rats were housed singly. Rats were maintained with free access to food and water under a $12: 12$ light/dark schedule at $22-24^{\circ} \mathrm{C}$ and $40 \%-60 \%$ humidity. Animals were first randomly assigned to one of four groups: pregnant CCI, non-pregnant CCI, pregnant sham, and non-pregnant sham, and then further randomly divided into two groups each for behavioral testing and tissue analysis.

\section{Pregnancy model}

The estrous cycle of the rats was monitored to determine the appropriate time for mating. For mating, one male and one female rat were placed into a cage. After mating, the rats were housed singly. The first day of mating in which a mucus plug was detected in the cage was designated as day 1 of pregnancy. CCI was performed on day 3 of pregnancy. All rats with a mucus plug were confirmed to be pregnant by either delivery or postmortem detection of a fetus in the uterus.

\section{$\mathrm{CCl}$ model}

Neuropathic pain was modeled by CCI in rats. The CCI procedure was performed as previously described. ${ }^{20}$ Rats were anesthetized by intraperitoneal injection of sodium pentobarbital $(50 \mathrm{mg} / \mathrm{kg})$. The common left sciatic nerve was exposed, and a 10-15 mm length of sciatic nerve proximal to the sciatic trifurcation was carefully dissected from the underlying tissue using blunt dissection through the biceps femoris. Four ligatures ( 4.0 braided silk) ${ }^{21}$ were tied loosely around the nerve at $1 \mathrm{~mm}$ intervals.

In the sham group, an identical dissection procedure was performed in the left hindpaw, but the sciatic nerve was not ligated. All surgical procedures were performed under strict sterile conditions.

\section{Mechanical threshold}

The mechanical threshold was determined using 10 calibrated von Frey filaments (Stoelting, Wood Dale, IL, USA) (0.4, $0.7,1.2,1.5,2.0,3.6,5.5,8.5,11.8$, and $15.1 \mathrm{~g}$ ) applied serially to only the left hindpaw in ascending order of strength. Behavioral tests were performed (pregnant sham and CCI group, $n=5$; non-pregnant Sham group, $n=6$; non-pregnant CCI group, $n=7$ ) at several time points (4 days before mating: baseline threshold, on the day of mating, and 7, 14, 21, and 28 days after confirming pregnancy; Figure 1). On the morning of the test day, the animals were placed in non-transparent plastic cubicles on a mesh floor for an acclimatization period of at least 30 minutes. A positive response was defined as rapid withdrawal and/or licking of the paw immediately upon application of the stimulus. Whenever a positive response to a stimulus occurred, the next smaller von Frey hair was applied, and whenever a negative response occurred, the next larger von Frey hair was applied. In the absence of a response at a pressure of $15.1 \mathrm{~g}$, the animal's response was assigned this cutoff value. The mechanical threshold was determined 


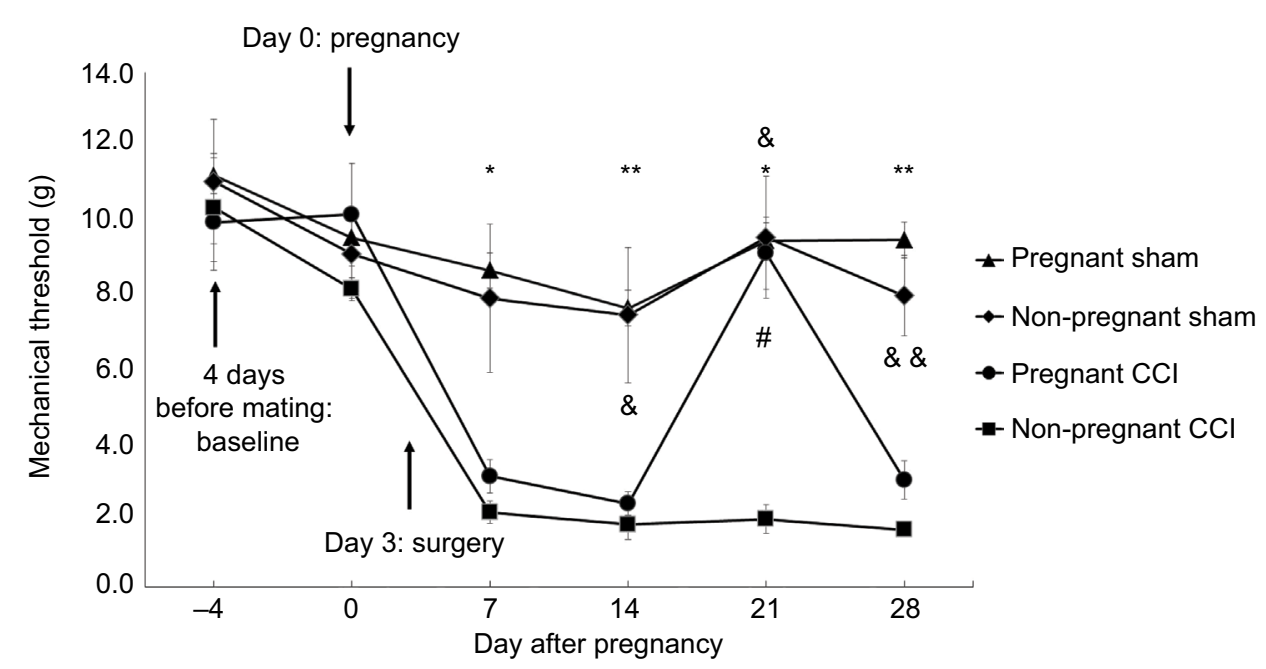

Figure I The anti-allodynic effect of pregnancy in a rat model of CCl. Arrows indicate the time of pregnancy and surgery. The time-course of the anti-allodynic effects of pregnancy in rats with $\mathrm{CCl}$. For comparison of the differences at individual time points between the four groups, we used Kruskal-Wallis and $D$ unn post hoc tests $* P<0.05$ pregnant sham vs non-pregnant $C C l$. ${ }^{* * P}<0.01$ pregnant sham vs non-pregnant $C C l$. ${ }^{\circledR} P<0.05$ non-pregnant sham vs non-pregnant $C C l$. ${ }^{\&} P<0.01$ non-pregnant sham vs nonpregnant $\mathrm{CCl}$. ${ }^{*}<<0.05$ pregnant $\mathrm{CCl}$ vs non-pregnant $\mathrm{CCl}$. Separate groups of rats were used for the behavioral test and tissue analysis.

Abbreviation: $\mathrm{CCl}$, chronic constriction injury.

according to the method described previously, with a tactile stimulus producing a $50 \%$ likelihood of withdrawal determined using the up-down method. ${ }^{22}$

\section{Western blot}

The L4/5 dorsal spinal cord was harvested at day 21 of pregnancy for the pregnant groups and 18 days after surgery for the non-pregnant groups. After deeply anesthetizing the rats ( $n=3$ /group), we extracted the spinal cord and divided it into the left and right halves. We then divided the left half into the ventral and dorsal halves. We used only the left dorsal side for the assays. The left L4-5 spinal dorsal horn (ie, ipsilateral to CCI surgery) was rapidly removed, frozen on dry ice, and stored at $-80^{\circ} \mathrm{C}$. The tissues were homogenized in protein lysis buffer (150 mM sodium chloride, 1.0\% NP-40, $0.5 \%$ sodium deoxycholate, $0.1 \%$ sodium dodecyl sulfate, and $50 \mathrm{mM}$ Tris, $\mathrm{pH} 8.0$ ) containing protease inhibitors and phosphatase inhibitors (phosphatase inhibitor cocktails 1/2). The homogenate was centrifuged at $18,000 \times g$ for $20 \mathrm{~min}$ utes at $4^{\circ} \mathrm{C}$. The supernatant was collected and assayed for protein concentration using the DC protein assay (Bio-Rad, Hercules, CA, USA). Aliquots containing $30 \mu \mathrm{g}$ protein were dissolved in LaemmLi buffer and denatured at $95^{\circ} \mathrm{C}$ for 5 minutes; the proteins were separated by $10 \%$ Tris-glycine sodium dodecyl sulfate-polyacrylamide gel electrophoresis gel and transferred to a polyvinylidene difluoride membrane. The membranes were blocked with 5\% nonfat dry milk in phosphate-buffered saline and then incubated with primary antibodies overnight at $4^{\circ} \mathrm{C}$, including rabbit anti-TNF- $\alpha$
(1:1000, Millipore Billerica, MA, USA), mouse anti-GFAP (1:5000, Sigma-Aldrich Co., St Louis, MO, USA), rabbit anti-Iba-1 (1:1000, Wako Pure Chemicals, Osaka, Japan), rabbit polyclonal anti c-Fos (1:1000, Santa Cruz Biotechnology, Dallas, TX, USA), and mouse anti- $\beta$-actin (1:5000, Sigma-Aldrich Co.). The blots were incubated with secondary antibodies (Santa Cruz Biotechnology) and developed in chemiluminescence solution (Pierce Biotechnology, Rockford, IL, USA). The Western blot results were quantified based on the obtained chemiluminescence values. Target protein bands were normalized to the amount of $\beta$-actin.

\section{Enzyme-linked immunosorbent assay}

Serum TNF- $\alpha$ concentrations were assessed by a double antibody sandwich enzyme-linked immunosorbent assay using a Quantikine ELISA Kit from R\&D Systems according to the manufacturer's instructions. Serum samples were diluted twofold with Calibrator Diluent RD5-17. The wells were read at $540 \mathrm{~nm}$ using Epoch2 (BioTek, Winooski, VT, USA). Each reaction was run in duplicate. The limit of sensitivity was $<12.5 \mathrm{pg} / \mathrm{mL}$, and the limit of linearity was $800 \mathrm{pg} / \mathrm{mL}$.

\section{Data analysis and statistics}

Statistical analysis was performed with GraphPad prism version 5 GraphPad Software (La Jolla, CA, USA). All values are expressed as mean \pm standard error of the mean (SEM). The behavioral responses to von Frey stimulation, indicating mechanical sensitivity, were used to indicate the mechanical threshold. The mechanical threshold results were evaluated 
by Kruskal-Wallis and Dunn post hoc tests. $P$-values $<0.05$ were considered statistically significant. The sample size estimate was based on our previous studies. ${ }^{23-25}$

Data of the effects of pregnancy on neurochemical changes were compared among the four groups with one-way analysis of variance (ANOVA) using the Tukey or Dunnett's post hoc test.

\section{Results}

\section{Development of neuropathic pain symptoms}

All rats with CCI of the sciatic nerve developed neuropathic pain symptoms as revealed by the von Frey test (Figure 1). Tactile hypersensitivity, ie, a decrease in mechanical threshold, was observed following CCI from day 7 through day 28 in the non-pregnant rats. The mechanical threshold was significantly higher in pregnant CCI rats on day 21 of pregnancy compared with the non-pregnant CCI group $(P=0.033)$. Comparison of the differences at individual time points among the four groups is shown in Figure 1. The anti-allodynic effect of pregnancy disappeared on day 28 .

\section{Effect of pregnancy on TNF- $\alpha$ expression in the spinal dorsal horn}

Sciatic nerve CCI leads to neuropathic pain-induced increases in TNF- $\alpha$ expression in the spinal cord. ${ }^{26}$ In the present study, we examined whether the increase in TNF- $\alpha$ expression induced by CCI-induced neuropathic pain was reduced during pregnancy.
TNF- $\alpha$ expression in the spinal dorsal horn was compared in the four groups using one-way ANOVA. TNF- $\alpha$ was significantly different among the four groups ( $F[3,8]=6.416$, $P=0.016)$. TNF- $\alpha$ expression was significantly higher in the non-pregnant CCI group compared with the non-pregnant sham group $(P=0.046)$. TNF- $\alpha$ expression was significantly lower in the pregnant-CCI group compared with the nonpregnant CCI group. There was no difference between the pregnant sham and the non-pregnant sham $(P=0.93$; Figure 2$)$.

\section{Expression of GFAP and Iba-I was modulated by pregnancy}

Previous findings demonstrated that spinal astrocytes and microglia make important mechanistic contributions to the initiation and maintenance of neuropathic pain. ${ }^{27}$ To examine whether nerve injury induced a glial reaction in the spinal dorsal horn, we observed changes in the expression of GFAP, a marker of reactive gliosis and proliferation of astrocytes, and Iba-1, a marker of microglia.

We used Western blot to quantify the differences in the GFAP levels in the spinal dorsal horn among the four groups (Figure 3). GFAP levels differed significantly among the four groups $(\mathrm{F}[3,8]=6.393, P=0.016)$. GFAP expression was significantly decreased in the pregnant CCI group compared to the non-pregnant CCI group $(P=0.014)$.

The same trend was observed for Iba-1 (Figure 4). Iba-1 levels were significantly different among the four groups (F $[3,8]=5.407, P=0.025)$. Iba-1 expression was significantly

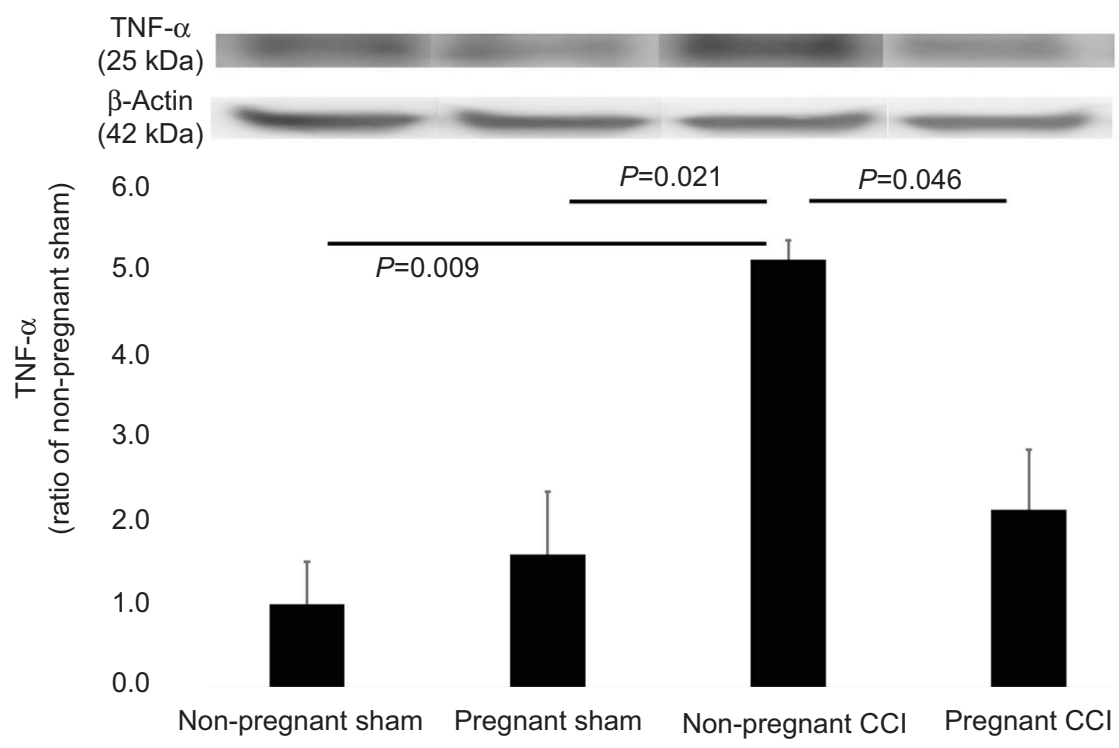

Figure 2 Expression of TNF- $\alpha$ in the L4-5 spinal dorsal horn mediated by pregnancy in a CCl model. The CCl surgical procedure increased the expression of TNF- $\alpha$ in the spinal dorsal horn of the non-pregnant $\mathrm{CCl}$ group compared with the non-pregnant sham groups. Expression of TNF- $\alpha$ was reduced in the pregnant $\mathrm{CCl}$ group compared with the non-pregnant $\mathrm{CCl}$ group. Data were analyzed using Dunnett's test, $\mathrm{n}=3$.

Abbreviations: $\mathrm{CCl}$, chronic constriction injury; TNF, tumor necrosis factor. 


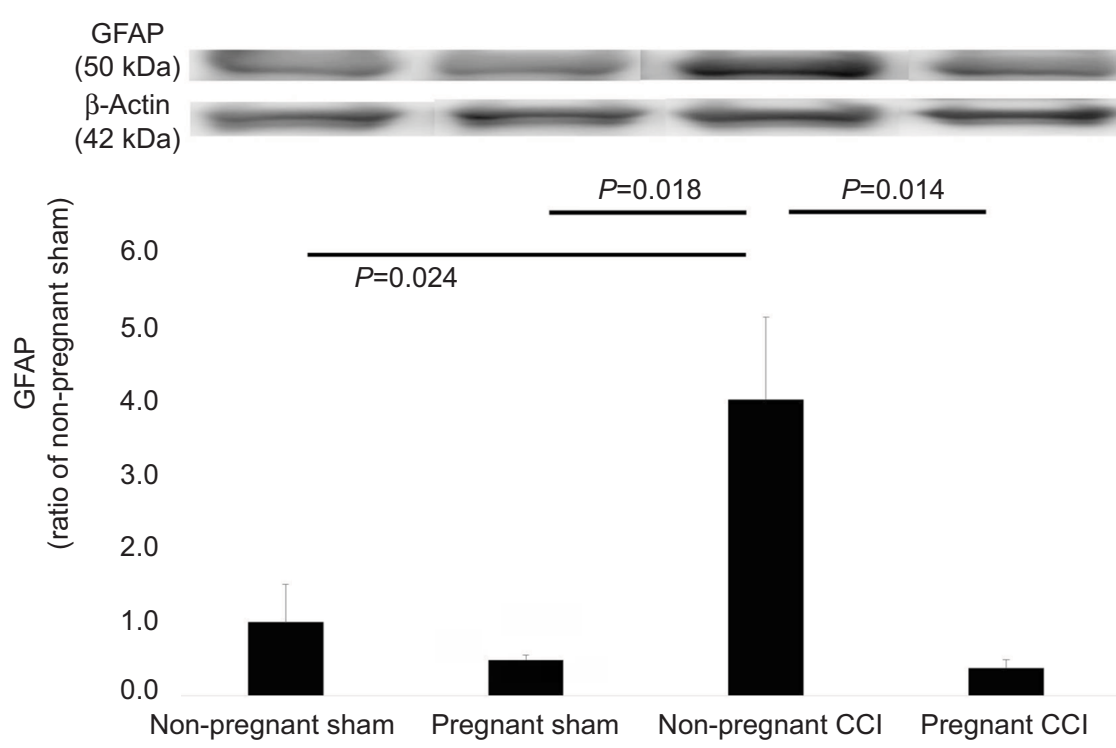

Figure 3 Expression of GFAP in the L4-5 spinal dorsal horn mediated by pregnancy in a CCI model. The CCl surgical procedure increased the expression of GFAP in the spinal dorsal horn of the non-pregnant $\mathrm{CCl}$ group compared with the non-pregnant sham groups. Expression of GFAP was reduced in the pregnant $\mathrm{CCl}$ group compared with the non-pregnant $\mathrm{CCl}$ group. Data were analyzed using Dunnett's test, $\mathrm{n}=3$ /group.

Abbreviations: $\mathrm{CCl}$, chronic constriction injury; GFAP, glial fibrillary acidic protein.

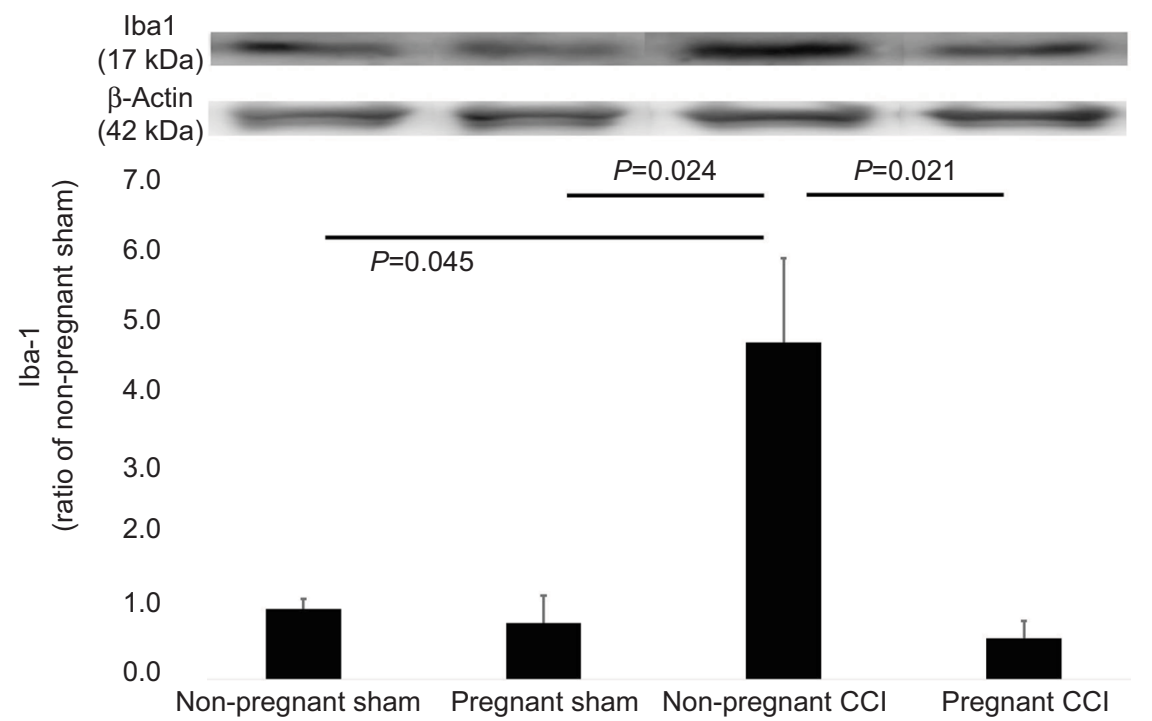

Figure 4 Expression of Iba- I in the L4-5 spinal dorsal horn mediated by pregnancy in a CCl model. The CCl surgical procedure increased the expression of Iba- I in the spinal dorsal horn of the non-pregnant $\mathrm{CCl}$ group compared with the non-pregnant sham groups. Expression of lba-I was reduced in the pregnant $\mathrm{CCl}$ group compared with the non-pregnant $\mathrm{CCl}$ group. Data were analyzed using Dunnett's test, $\mathrm{n}=3$ /group.

Abbreviation: $\mathrm{CCl}$, chronic constriction injury.

decreased in the pregnant CCI group compared to the nonpregnant CCI group $(P=0.021)$.

Consistent with behavioral allodynia in the non-pregnant CCI group (Figure 1) compared with the pregnant CCI group on day 21, an increase in GFAP and Iba-1 expression was observed in the spinal dorsal horn at the same time point. The CCI-induced increases were suppressed by pregnancy. GFAP and Iba-1 expression levels were not significantly different between the pregnant sham and the non-pregnant sham in $\operatorname{GFAP}(P=0.997)$ and $\operatorname{Iba}-1(P=0.973)$.

\section{Expression of c-Fos in the spinal dorsal horn was reduced by pregnancy}

c-Fos is a commonly used molecular marker of enhanced neuronal activity in the pain neural pathway. ${ }^{28}$ In this study, we investigated whether the expression of c-Fos protein in 


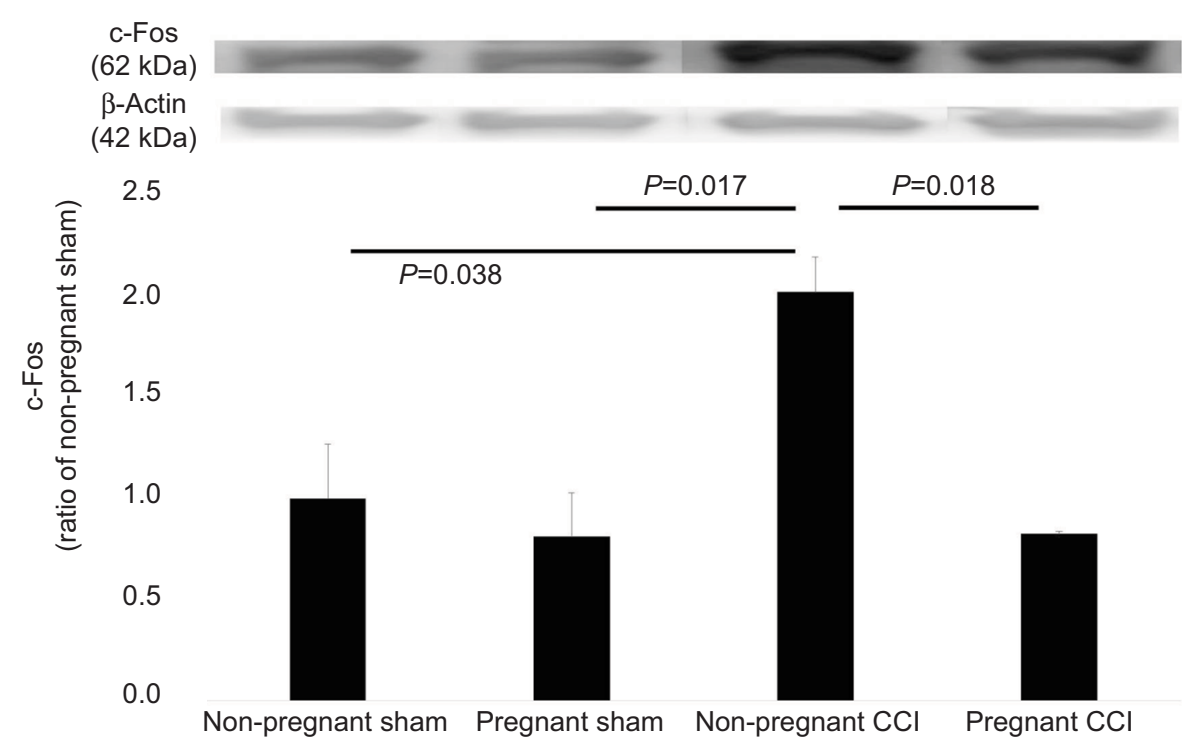

Figure 5 Expression of c-Fos in the L4-5 spinal dorsal horn mediated by pregnancy in a $\mathrm{CCl}$ model. The $\mathrm{CCl}$ surgical procedure increased the expression of c-Fos in the spinal dorsal horn of the non-pregnant $\mathrm{CCl}$ group compared with the non-pregnant sham groups. Expression of lba- $\mathrm{I}$ was reduced in the pregnant $\mathrm{CCl}$ group compared with the non-pregnant $\mathrm{CCl}$ group. Data were analyzed using Dunnett's test, $\mathrm{n}=3$ /group.

Abbreviation: $\mathrm{CCl}$, chronic constriction injury.

the spinal dorsal horn was decreased within 1 day before parturition in a rat model of CCI. C-Fos protein was measured using Western blot (Figure 5). C-Fos levels were significantly different among the four groups ( $\mathrm{F}[3,8]=5.965, P=0.019)$. The expression of c-Fos was decreased in the pregnant CCI group compared with the non-pregnant $\mathrm{CCI}$ group $(P=0.018)$. There were no differences between the pregnant sham and the non-pregnant sham in c-Fos $(P=0.939)$.

\section{Serum TNF- $\alpha$ levels}

Although we attempted to measure serum TNF- $\alpha$ in the four groups on days 0 and 21, it was not detected (data not shown).

\section{Discussion}

The findings of the present study demonstrated that pregnancy-induced analgesia suppresses neuropathic pain due to $\mathrm{CCI}$ in rats and decreases the expression of TNF- $\alpha$ in the spinal cord. The behavioral results indicated that the mechanical thresholds of pregnant rats and non-pregnant rats were approximately equal during the first 18 days after CCI surgery, but on day 21 after pregnancy, pregnant CCI rats exhibited anti-allodynia compared with the non-pregnant CCI rats. After delivery, the anti-allodynic effects of pregnancy disappeared. The findings also revealed that TNF- $\alpha$ expression in CCI rats was decreased within 1 day before parturition. Further, pregnancy decreased the CCI-induced increase expression of GFAP, Iba-1, and c-Fos in the spinal dorsal horn. There is no difference between non-pregnant sham to pregnant sham. I think that TNF- $\alpha$, GFAP, and Iba1 , which were elevated by CCI, are reduced by pregnancy. TNF- $\alpha$, GFAP, and Iba- 1 do not decrease even if rats who do not have pain when become pregnant. Serum TNF- $\alpha$ was below the detection limits in all groups.

Pregnancy induces analgesia against somatic and visceral pain just before parturition. ${ }^{8,10}$ In laboratory animals, pregnancy-induced antinociception is due to the contribution of both peripheral ${ }^{29}$ and central processes. ${ }^{30}$ We focused on neuropathic pain because the effect of pregnancy on neuropathic pain is not clear. While some reports indicate that women experience a progressive increase in the heat pain threshold during pregnancy, ${ }^{12}$ others do not. ${ }^{13}$ These conflicting phenomena indicate that pregnancy does not induce analgesia in all individuals. ${ }^{8}$ In general, gonadotropins gradually increase during pregnancy and decrease immediately after delivery. ${ }^{31}$ In rats, pregnancy-induced analgesia is reported to peak abruptly on day $21 .{ }^{10}$ Our findings also demonstrated an abrupt peak in pregnancy-induced analgesia on day 21 . Gintzler reported that endogenous opioids are associated with pregnancy-induced analgesia. ${ }^{10}$ For these reasons, we speculate that abrupt pregnancy-induced analgesia just prior to parturition was generated from not only gonadotropins, but also other endogenous substances, including endogenous opioids.

TNF- $\alpha$ is a pleiotropic cytokine produced mainly by activated macrophages and $\mathrm{T}$ cells in response to inflammation and by mast and Schwann cells in response to peripheral 
nerve injury. ${ }^{26}$ The most common pathogenic mechanism of peripheral neuropathies is a pathway involving proinflammatory cytokines such as TNF- $\alpha$ that increase vascular permeability, causing a breakdown of the vascular wall, which permits leukocyte transmigration into the nerves. ${ }^{32}$ This induces the activation and proliferation of lymphocytes and macrophages, which have direct neurotoxic activity, and further the release of cytokines such as TNF- $\alpha .{ }^{32}$ Evidence indicates that peripheral nerve damage or inflammation activates glia in the dorsal horn, which plays an important role in the pathogenesis of neuropathic pain. ${ }^{33}$ After peripheral nerve injury or spinal cord injury, ${ }^{17}$ TNF- $\alpha$ levels in spinal astrocytes are increased. ${ }^{34}$ Previous studies demonstrated that overexpression of spinal TNF- $\alpha$ released from microglia and/or astrocytes plays an important role in the different neuropathic pain models. ${ }^{35}$ Microglia are also an important therapeutic target for neuropathic pain. ${ }^{36}$ Microglial membrane adaptor protein was recently reported to be involved in the development of neuropathic pain. ${ }^{37}$ Microglia are related to the initiation of hypersensitivity and the transition from acute to chronic pain after peripheral nerve injury, working synergistically with monocytes. ${ }^{38}$ The present study showed that GFAP and Iba-1 levels were also reduced when TNF- $\alpha$ release was suppressed. The activity of astrocytes and microglia might be inhibited by pregnancy, thereby reducing the secretion of TNF- $\alpha$. Previous findings indicated that serum TNF- $\alpha$ is not detected in pregnant rats. ${ }^{39} \mathrm{We}$ also found that the serum TNF- $\alpha$ was below the detection limits in all groups, whereas TNF- $\alpha$ at the spinal dorsal horn was detected in all groups, and suppressed by pregnancy. These findings indicate that the decrease in TNF- $\alpha$ at the spinal dorsal horn plays an important role in pregnancy-induced analgesia.

As one of the immediate early genes, c-Fos and its protein product FOS are expressed in cells rapidly and transiently after various forms of stimulation. ${ }^{40}$ Sensory input, especially nociceptive input, increases the expression of FOS..$^{28,41,42}$ This increase in FOS reflects postsynaptic excitation of dorsal horn neurons, which is mediated by primary afferent input (monosynaptic) or through the large dorsal horn interneuronal pools of glutamatergic neurons with excitatory linkages mediated by a variety of glutamate receptors, including those of the N-methyl-D-aspartate and $\alpha$-amino-3-hydroxy-5-methyl-4isoxazole propionic acid subtypes. ${ }^{43}$ As mentioned above, these findings suggest that pregnancy reduces the excitation of neurons that exhibit c-Fos expression.

An effective treatment for neuropathic pain is in high demand. Understanding the underlying mechanism of pregnancy-induced analgesia could potentially elucidate new therapeutic targets to pursue. Research examining this possibility has revealed beneficial effects of $17 \mathrm{~b}$-estradiol on neuropathic pain and neuronal regeneration. ${ }^{44}$ Moreover, Huang et al reported that anti-TNF- $\alpha$ therapy using a herpes simplex virus vector, or gene therapy, suppresses human immunodeficiency virus-related neuropathic pain..$^{45}$ These findings support the idea that treatment with gonadal hormones and TNF- $\alpha$ antagonist should be considered as a potential therapeutic target.

\section{Conclusion}

In summary, pregnancy-induced analgesia suppresses neuropathic pain due to $\mathrm{CCI}$ in rats and decreases the expression of TNF- $\alpha$ in the spinal cord. Activities of astrocytes and microglia are suppressed during pregnancy. Suppression of neuronal activity in the spinal dorsal horn is critical for pregnancy-induced analgesia against neuropathic pain. Further research using an animal model of neuropathic pain during pregnancy may help to clarify the interaction between TNF- $\alpha$ and GFAP/Iba-l and gonadotropin, which could potentially lead to the development of novel treatments against human neuropathic pain.

\section{Acknowledgments}

This work was supported by JSPS KAKENHI Grant Numbers, JP21591994, JP24791577, JP16K20077, and JP15K20026. The authors thank Dr Shuanglin Hao and Dr Keiko Mamiya for technical help and appropriate advice.

\section{Disclosure}

The authors report no conflicts of interest in this work.

\section{References}

1. Woolf CJ, Mannion RJ. Neuropathic pain: aetiology, symptoms, mechanisms, and management. Lancet. 1999;353(9168):1959-1964.

2. Torrance N, Ferguson JA, Afolabi E, et al. Neuropathic pain in the community: more under-treated than refractory? Pain. 2013;154(5):690-699.

3. Rice ASC, Hill RG. New treatments for neuropathic pain. Annu Rev Med. 2006;57:535-551.

4. Finnerup NB, Attal N, Haroutounian S, et al. Pharmacotherapy for neuropathic pain in adults: a systematic review and meta-analysis. Lancet Neurol. 2015;14(2):162-173.

5. Gallagher RM. Management of neuropathic pain: translating mechanistic advances and evidence-based research into clinical practice. Clin J Pain. 2006;22(1 Suppl):S2-S8.

6. Eisenberg E, McNicol ED, Carr DB. Efficacy and safety of opioid agonists in the treatment of neuropathic pain of nonmalignant origin: systematic review and meta-analysis of randomized controlled trials. JAMA. 2005;293(24):3043-3052.

7. Edelsberg J, Oster G. Summary measures of number needed to treat: How much clinical guidance do they provide in neuropathic pain? Eur J Pain. 2009;13(1):11-16.

8. Iwasaki H, Collins JG, Saito Y, Kerman-Hinds A. Naloxone-sensitive, pregnancy-induced changes in behavioral responses to colorectal distention: pregnancy-induced analgesia to visceral stimulation. Anesthesiology. 1991;74(5):927-933. 
9. Gupta DS, Gintzler AR. Influence of ovarian sex steroids on spinal methionine-enkephalin release: comparison with dynorphin reveals asymmetrical regulation. J Pharmacol Exp Ther. 2003;304(2):738-744.

10. Gintzler AR. Endorphin-mediated increases in pain threshold during pregnancy. Science. 1980;210(4466):193-195.

11. Jarvis S, McLean KA, Chirnside J, et al. Opioid-mediated changes in nociceptive threshold during pregnancy and parturition in the sow. Pain. 1997;72(1-2):153-159.

12. Draisci G, Catarci S, Vollono C, et al. Pregnancy-induced analgesia: a combined psychophysical and neurophysiological study. Eur J Pain (United Kingdom). 2012;16(10):1389-1397.

13. Frölich MA, Banks C, Warren W, Robbins M, Ness T. The association between progesterone, estradiol, and oxytocin and heat pain measures in pregnancy. Anesth Analg. 2016;123(2):396-401.

14. Carvalho B, Granot M, Sultan P, Wilson H, Landau R. A longitudinal study to evaluate pregnancy-induced endogenous analgesia and pain modulation. Reg Anesth Pain Med. 2016;41(2):175-180.

15. Sorkin LS, Doom CM. Epineurial application of TNF elicits an acute mechanical hyperalgesia in the awake rat. J Peripher Nerv Syst. 2000; 5(2):96-100.

16. Zheng WW, Ouyang HD, Zheng XX, et al. Glial TNFalpha in the spinal cord regulates neuropathic pain induced by HIV gp120 application in rats. Mol Pain. 2011;7(1):40.

17. Peng X, Zhou Z, Glorioso JC, Fink DJ, Mata M. Tumor necrosis factoralpha contributes to below-level neuropathic pain after spinal cord injury. Ann Neurol. 2006;59(5):843-851.

18. Deleo JA, Colburn RW, Rickman AJ. Cytokine and growth factor immunohistochemical spinal profiles in two animal models of mononeuropathy. Brain Res. 1997;759(1):50-57.

19. Zimmermann M. Ethical guidelines for investigations of experimental pain in conscious animals. Pain. 1983;16(2):109-110.

20. Bennett GJ, Xie YK. A peripheral mononeuropathy in rat that produces disorders of pain sensation like those seen in man. Pain. 1988;33(1): 87-107.

21. van der Wal S, Cornelissen L, Behet M, Vaneker M, Steegers M, Vissers $\mathrm{K}$. Behavior of neuropathic pain in mice following chronic constriction injury comparing silk and catgut ligatures. Springerplus. 2015;4:225.

22. Chaplan SR, Bach FW, Pogrel JW, Chung JM, Yaksh TL. Quantitative assessment of tactile allodynia in the rat paw. $J$ Neurosci Methods. 1994;53(1):55-63.

23. Kanda H, Liu S, Iida T, et al. Inhibition of mitochondrial fission protein reduced mechanical allodynia and suppressed spinal mitochondrial superoxide induced by perineural human immunodeficiency virus gp120 in rats. Anesth Analg. 2016;122(1):264-272.

24. Kanda H, Kanao M, Liu S, et al. HSV vector-mediated GAD67 suppresses neuropathic pain induced by perineural HIV gp120 in rats through inhibition of ROS and Wnt5a. Gene Ther. 2016;23(4):340-348.

25. Kanao M, Kanda H, Huang W, et al. Gene transfer of glutamic acid decarboxylase 67 by herpes simplex virus vectors suppresses neuropathic pain induced by human immunodeficiency virus gp 120 combined with ddC in rats. Anesth Analg. 2015;120(6):1394-1404.

26. Shubayev VI, Myers RR. Upregulation and interaction of TNF $\alpha$ and gelatinases A and B in painful peripheral nerve injury. Brain Res. 2000;855(1):83-89.

27. DeLeo JA, Yezierski RP. The role of neuroinflammation and neuroimmune activation in persistent pain. Pain. 2001;90(1-2):1-6.

Journal of Pain Research

\section{Publish your work in this journal}

The Journal of Pain Research is an international, peer reviewed, open access, online journal that welcomes laboratory and clinical findings in the fields of pain research and the prevention and management of pain. Original research, reviews, symposium reports, hypothesis formation and commentaries are all considered for publication.
28. Hunt SP, Pini A, Evan G. Induction of c-fos-like protein in spinal cord neurons following sensory stimulation. Nature. 1987;328(6131):632-634.

29. Dawson-Basoa MB, Gintzler AR. 17- $\beta$-Estradiol and progesterone modulate an intrinsic opioid analgesic system. Brain Res. 1993;601(1-2): 241-245.

30. Dawson-Basoa M, Gintzler AR. Gestational and ovarian sex steroid antinociception: synergy between spinal $\kappa$ and $\delta$ opioid systems. Brain Res. 1998;794(1):61-67.

31. Bridges RS. A quantitative analysis of the roles of dosage, sequence, and duration of estradiol and progesterone exposure in the regulation of maternal behavior in the rat. Endocrinology. 1984;114(3):930-940.

32. Sykam A, Gutlapalli VR, Tenali SP, et al. Association of tumor necrosis factor-alpha and interferon gamma gene polymorphisms and their plasma levels in leprosy, HIV and other peripheral neuropathies. Cytokine. 2015;76(2):473-479.

33. Myers RR, Shubayev VI. The ology of neuropathy: an integrative review of the role of neuroinflammation and TNF-alpha axonal transport in neuropathic pain. J Peripher Nerv Syst. 2011;16(4):277-286.

34. Raghavendra V, Rutkowski MD, DeLeo JA. The role of spinal neuroimmune activation in morphine tolerance/hyperalgesia in neuropathic and sham-operated rats. J Neurosci. 2002;22(22):9980-9989.

35. Wei F, Guo W, Zou S, Ren K, Dubner R. Supraspinal glial-neuronal interactions contribute to descending pain facilitation. $J$ Neurosci. 2008;28(42):10482-10495.

36. Tsuda M, Shigemoto-Mogami Y, Koizumi S, et al. P2X4 receptors induced in spinal microglia gate tactile allodynia after nerve injury. Nature. 2003;424(6950):778-783.

37. Guan Z, Kuhn JA, Wang X, et al. Injured sensory neuron-derived CSF1 induces microglial proliferation and DAP12-dependent pain. Nat Neurosci. 2015;19:1-10.

38. Peng J, Gu N, Zhou L, et al. Microglia and monocytes synergistically promote the transition from acute to chronic pain after nerve injury. Nat Commun. 2016;7:12029.

39. Caja S, Puerta M. White adipose tissue production and release of IL-6 and TNF- $\alpha$ do not parallel circulating and cerebrospinal fluid concentrations in pregnant rats. Horm Metab Res. 2008;40(6):375-380.

40. Harris JA. Using c-fos as a neural marker of pain. Brain Res Bull. 1998; 45(1):1-8.

41. Smits H, Kleef MV, Honig W, Gerver J, Gobrecht P, Joosten EAJ. Spinal cord stimulation induces c-Fos expression in the dorsal horn in rats with neuropathic pain after partial sciatic nerve injury. Neurosci Lett. 2009;450(1):70-73.

42. Gao Y-J, Ji R-R. c-Fos and pERK, which is a better marker for neuronal activation and central sensitization after noxious stimulation and tissue injury? Open Pain J. 2009;2(Suppl 2):11-17.

43. Zhang E-T, Ossipov MH, Zhang D-Q, Lai J, Porreca F. Nerve injuryinduced tactile allodynia is present in the absence of FOS labeling in retrogradely labeled post-synaptic dorsal column neurons. Pain. 2007;129(1-2):143-154.

44. Vacca V, Marinelli S, Pieroni L, Urbani A, Luvisetto S, Pavone F. 17beta-estradiol counteracts neuropathic pain: a behavioural, immunohistochemical, and proteomic investigation on sex-related differences in mice. Sci Rep. 2016;6(2):18980.

45. Huang W, Zheng W, Liu S, et al. HSV-mediated p55TNFSR reduces neuropathic pain induced by HIV gp120 in rats through CXCR4 activity. Gene Ther. 2014;21(3):328-336.

The manuscript management system is completely online and includes a very quick and fair peer-review system, which is all easy to use. Visit http://www.dovepress.com/testimonials.php to read real quotes from published authors. 